\title{
Construction autophagy-related prognostic risk signature to facilitate survival prediction, individual treatment and biomarker excavation of epithelial ovarian cancer patients
}

\author{
Hongjun Fei ${ }^{1+}$, Songchang Chen ${ }^{1,2+}$ and Chenming $\mathrm{Xu}^{1,2^{*}}$
}

\begin{abstract}
Background: Existing clinical methods for prognosis evaluating for Epithelial Ovarian Cancer (EOC) patients had defects of invasive, unsystematic and subjective and little data are available for individualizing treatment, therefore, to identify potential prognostic markers and new therapeutic targets for EOC is urgently required.

Results: Expression of 232 autophagy-related genes (ARGs) in 354 EOC and 56 human ovarian surface epithelial specimens from 7 independent laboratories were analyzed, 31 mRNAs were identified as DEARGs. We did functional and pathway enrichment analysis and constructed protein-protein interaction network for all DEARGs. To screen out candidate DEARGs related to EOC patients' survival and construct an autophagy-related prognostic risk signature, univariate and multivariate Cox proportional hazards models were established separately. Finally, 5 optimal independent prognostic DEARGS (PEX3, DNAJB9, RB1, HSP9OAB1 and CXCR4) were confirmed and the autophagy-related risk model was established by the 5 prognostic DEARGs. The accuracy and robustness of the prognostic risk model for survival prediction were evaluated and verified by analyzing the correlation between EOC patients' survival status, clinicopathological features and risk scores.

Conclusions: The autophagy-related prognostic risk model can be independently used to predict overall survival in EOC patients, it can also potentially assist in individualizing treatment and biomarker development.
\end{abstract}

Keywords: Epithelial ovarian Cancer, Autophagy-related genes, Prognostic risk model, Targeted therapeutic intervention, Survival prediction

\footnotetext{
*Correspondence: chenming_xu2006@163.com

${ }^{\dagger}$ Hongjun Fei and Songchang Chen contributed equally to this work.

'Department of Reproductive Genetics, International Peace Maternity and

Child Health Hospital, Shanghai Key Laboratory of Embryo Original Diseases,

Shanghai Municipal Key Clinical Specialty, Shanghai Jiao Tong University

School of Medicine, No.910, Hengshan Road, Shanghai 200030, People's

Republic of China

${ }^{2}$ Obstetrics and Gynecology Hospital of Fudan University, Shanghai 200011,

China
}

(c) The Author(s). 2021 Open Access This article is licensed under a Creative Commons Attribution 4.0 International License, which permits use, sharing, adaptation, distribution and reproduction in any medium or format, as long as you give appropriate credit to the original author(s) and the source, provide a link to the Creative Commons licence, and indicate if changes were made. The images or other third party material in this article are included in the article's Creative Commons licence, unless indicated otherwise in a credit line to the material. If material is not included in the article's Creative Commons licence and your intended use is not permitted by statutory regulation or exceeds the permitted use, you will need to obtain permission directly from the copyright holder. To view a copy of this licence, visit http://creativecommons.org/licenses/by/4.0/ The Creative Commons Public Domain Dedication waiver (http://creativecommons.org/publicdomain/zero/1.0/) applies to the data made available in this article, unless otherwise stated in a credit line to the data. 


\section{Introduction}

Ovarian cancer $(\mathrm{OC})$ has the highest morbidity and mortality in the female genital tract [1]. It is the fifth most frequent cause of cancer death in women in the United States in 2020 which results in the death of 5\% women with cancer, and 5-year survival rates are 39$48 \%$ of all the women diagnosed with ovarian cancer [2, 3]. Epithelial ovarian cancer (EOC) is the most common type of OC which accounts for almost $90 \%$ of all ovarian cancers $[4,5]$. It generally presents at an advanced stage in over $70 \%$ of patients contributing to a high death rate, where the long term survival rate ( 10 years) is estimated at $15-30 \%[6,7]$. The prognosis of EOC is associated with many factors such as histological type, pathological stage, age, early recognition, the volume of ascites and so on [8, 9], existing clinical methods for prognosis evaluation still had defects of invasive, unsystematic and subjective. It is necessary to refine the prognostic model of EOC and establish a more accurate method to managing this high-mortality disease.

Establishing prognosis models for EOC patients is an important part of risk evaluation and treatment, can also greatly assists in biomarker development [10]. From clinically applicability, prognosis model is a handy tool to estimate overall survival and risk of recurrence, and can potentially help in individualizing treatment for patients [11]; From research development, it can contribute to identify subgroups of patients with unfavorable prognosis and promote us to explore alternative treatment strategies for these patients, and provide an idea for targeting therapy [12]. The present study focuses on constructing a prognosis model for EOC by molecular typing methods using large databases.

Autophagy is a homeostatic mechanism that can maintain cell survival by recycling organelles and macromolecules [13]. Various genes named because of participating in autophagy, they were called Autophagy-Related Genes (ARGs) [14]. Accumulating data suggest that autophagy dysregulation in EOC cells caused dormancy and chemo -/radio-therapy resistance, and the process involving proteins (mainly ARGs encoding proteins) are being considered as anticancer molecular therapeutic targets
[15-18]. However, no prior study used the large-scale expression patterns for assessing the autophagy effect on EOC prognosis. So, this study makes use of ARGs to construct the prognostic risk signature of EOC. Through this novel prognostic risk model, we expect to shed light on prognosis evaluation and targeted treatment of EOC.

\section{Materials and methods}

\section{Data acquisition}

The gene expression profiling data sets (ID: GSE14407, GSE6008, GSE14001, GSE26712, GSE29450, GSE38666, GSE105437) were obtained from Gene Expression Omnibus database (https://www.ncbi.nlm.nih.gov/geo/). The brief information of 7 GEO datasets [containing 410 human ovarian surface epithelial (HOSE) and epithelial ovarian cancer (EOC) specimens] from 7 independent laboratories was extracted and listed in Table 1. The RNA-seq data and the corresponding clinical data of 379 EOC patients were downloaded from the TCGA database (https://portal.gdc.cancer.gov/). We downloaded 232 genes identified so far to be involved in autophagy from the Human Autophagy Database (HADb).

\section{Differentially expressed autophagy-related genes (DEAR Gs) screening}

Data preprocessing was performed before difference analysis for 7 independent GEO datasets. We did batch normalization for all expression profiling data through ComBat algorithm in $\mathrm{R}$ to eliminate the systematic variations among different studies. The differentially expressed autophagy-related genes (DEARGs) between 354 EOC tissues and 56 HOSE tissues were screened out by the Wilcoxon signed-rank test. The cutoff criteria were adjusted $p$-value $<0.05$ and $\mid \log _{2}$ FoldChange $\mid\left(\left|\log _{2} \mathrm{FC}\right|\right)>1$.

\section{Protein-protein interaction (PPI) network construction for all DEARGs}

PPI analysis is a protein correlation analysis that can effectively reveal the molecular mechanisms of crucial cellular activities in carcinogenesis. It is constructed based on the STRING database (https://string-db.org/). The PPI network was constructed for all DEARGs and

Table 1 Characteristic of microarray data used to do difference analysis

\begin{tabular}{llll}
\hline Expression profiling array (HOSE \& EOC) & Platforms & GEO accession & Samples \\
\hline Genome & GPL570 & GSE14407 & 12HOSE; 12EOC \\
& & GSE14001 & 3HOSE; 20EOC \\
& & GSE29450 & 10HOSE; 10EOC \\
& & GSE38666 & 12HOSE; 18EOC \\
Genome & GPL96 & GSE105437 & 5HOSE; 10EOC \\
& & GSE6008 & 4HOSE; 99EOC \\
\hline
\end{tabular}


visualized with the cut-off criterion of interaction score > 0.4. To visualized the PPI network and highlighted the hub genes, we make use of the Cytoscape software to perform deeply biological network analysis.

\section{Functional and pathway enrichment analysis for all DEAR Gs}

Gene Ontology (GO) analysis can annotate characteristics of a set of genes, such as involved cellular components (CC), molecular functions (MF) and biological processes (BP). Kyoto Encyclopedia of Genes and Genomes (KEGG) analysis was used to reveal the involved pathways of all DEARGs. These analyses were done by clusterProfiler package of $\mathrm{R}$ with $p$ value $<0.05$ as a strict cutoff.

\section{Identify the prognostic DEARGs}

To identify DEARGs whose expression profiles had a significant correlation with the overall survival (OS) of patients with EOC, we performed the univariate Cox regression model. The DEARGs with the threshold of $P<$ 0.05 were regarded as candidate genes related to EOC patients' survival.

\section{Construction of OS risk prognostic model and risk score calculation}

The survival-related DEARGs screened out through the univariate Cox proportional hazards model were incorporated into a multivariate Cox regression analysis to weeded out the DEARGs which might not be an independent index in prognosis monitoring. Then, we can obtain a set of optimal prognostic DEARGs, and establish a risk score model using these genes.

We can get the risk score for each EOC patient through the followed formula,

$$
\begin{aligned}
& \text { the risk score }=\sum_{\mathrm{i}=1,2, \ldots, \mathrm{n}} \text { regression coeffiecient }(\text { genei }) \\
& \times \text { expression value of (genei) }
\end{aligned}
$$

The risk score was calculated using the gene expression value multiplied by the regression coefficients of each individual gene. The genes in the formula mean the independent prognostic DEARGs we screened before. The regression coefficients were obtained from the multivariate Cox regression model and stand for the relative weight of selected genes. It is a measure of prognostic risk for EOC patients. With the median risk score as the cutoff value, patients were divided into low-risk group and high-risk group. A high-risk score means a poor prognosis.

\section{Evaluation of OS risk prognostic model}

We draw the survival curves by Kaplan-Meier plotter and assessed the difference in the survival status between the high-risk group and low-risk groups to verify the validity and robustness of the OS risk prognostic model. Then, the relationship between risk score and survival status of EOC patients was visualized. We studied genetic alterations of 5 independent prognostic DEARGs (PEX3, DNAJB9, RB1, HSP9OAB1 and CXCR4) in an Ovarian Cystadenocarcinoma case set using an open-access tool cBioPortal (http://www.cbioportal.org).

What's more, we performed Cox proportional hazard regression to evaluate whether the OS risk prognostic model constructed with DEARGs could independent of other clinicopathological features as a predictor of OS for EOC patients. The correlation between the risk score and clinical traits was analyzed by univariate and multivariate Cox regression methods. Age, pathological stage and histological grade were used as candidate clinicopathological risk factors. We want to explore whether clinicopathological risk factors and risk scores calculated based on prognostic DEARGs can all be indicators in prognosis monitoring for EOC patients, and to confirm whether the risk score could be an independent prognosis factor.

\section{Results \\ Identification of differentially expressed autophagy- related genes (DEARGs)}

The expression values of 13,045 genes in 410 samples (354 EOC and 56 HOSE specimens) were normalized with $\mathrm{R}$ package and showed in Fig. 1a. Horizontal axis and vertical axis represents 410 samples and all gene expression value, respectively. After batch normalization, the median of expression value of samples is almost the same, proved normalized data were qualified. The expression values of 232 ARGs were extracted. Considering adjust $p$-value $<0.05$ and $\left|\log _{2} \mathrm{FC}\right|>1$ as the filter criteria, 31 DEARGs (14 downregulated ARGs and 17 upregulated ARGs) were obtained. We list the details such as $\log _{2}$ FoldChange and statistical significance of all DEARGs in Table 2. In Fig. 1b, the fold change patterns of 31 DEARGs were showed in a heat map. The expression of 31 DEARGs between EOC tissues and HOSE tissues was visualized by scatter plots (Fig. 1c).

\section{PPI network construction and functional annotation of DEARGs}

We displayed the distribution of all DEARGs with a volcano plot (Fig. 2a). A PPI network of DEARGs was constructed with STRING database and visualized the interaction of the PPI network using Cytoscape software, there are 12 hub genes with interaction degree $>5$ showed as a diamond and arranged in a circle (Fig. 2b). 


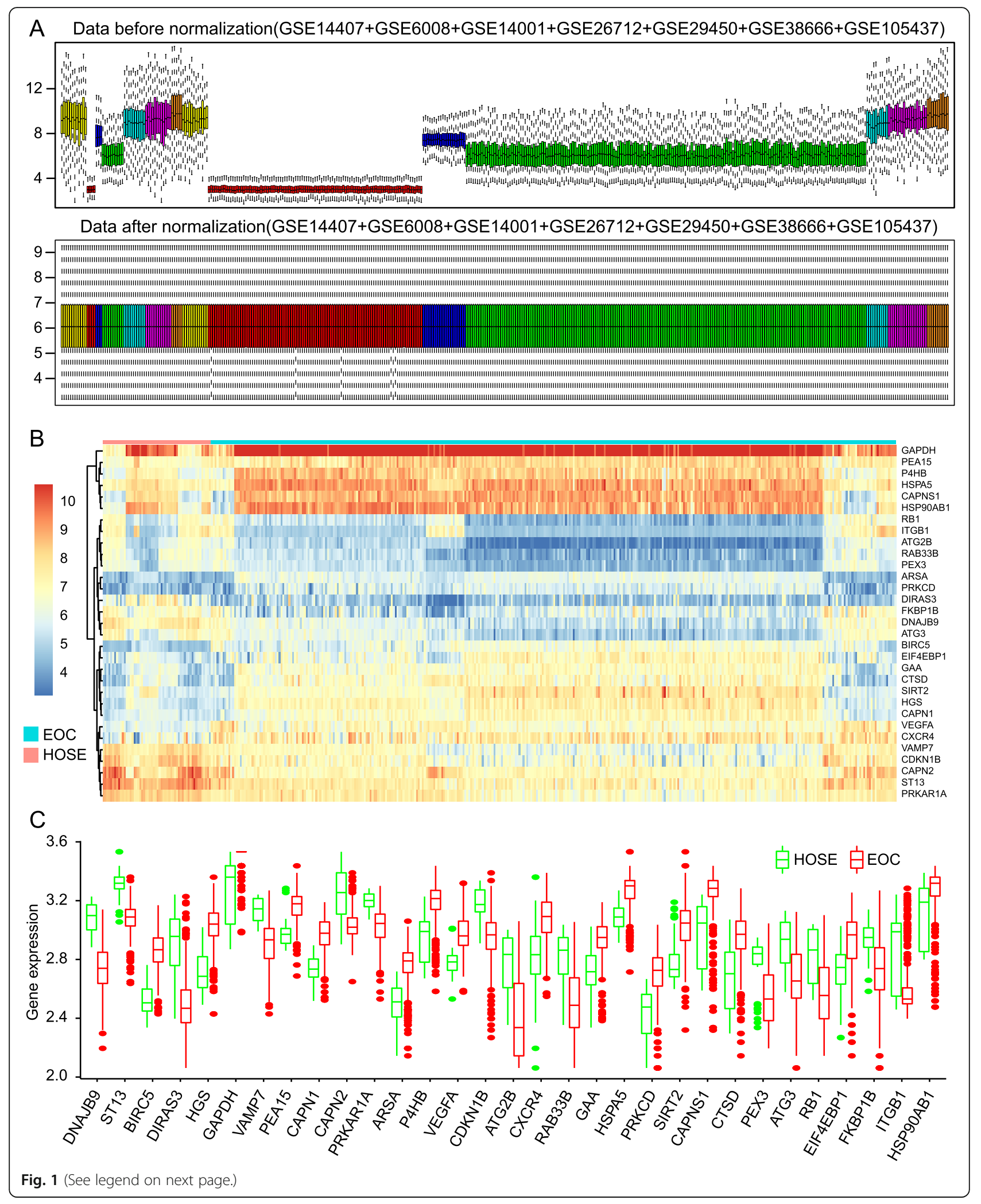


(See figure on previous page.)

Fig. 1 Differentially expressed autophagy-related genes (DEARGs) screened from 354 EOC tissues and 56 HOSE tissues. a The expression values of all genes before and after normalization were displayed by box Figs. X-axis represents 410 tissues samples from GSE14407, GSE6008, GSE14001, GSE26712, GSE29450, GSE38666, and GSE105437 datasets. The 7 groups on the left were HOSE tissues, and the right were EOC tissues. The same color columns represent samples that came from the same GEO datasets. $\mathbf{b}$ Heatmap of the expression levels of 31 DEARGs in EOC. The depth of blue and red color represents the intensity of the expression level of DEARGs. EOC, Epithelial Ovarian Cancer. c Visualization of expression patterns of 31 DEARGs. Red box plots represent EOC samples and green represent HOSE samples

Table 2 All DEARGs, screened between human ovarian surface epithelia (HOSE) tissues and epithelial ovarian cancer (EOC) tissues with criteria of adjust- $p$-Value $<0.05$ and | $\log _{2}$ FoldChange| $>1$

\begin{tabular}{|c|c|c|c|}
\hline Gene & $\log _{2} F C$ & $p$-Value & $\overline{\text { adjust- } p \text {-Value }}$ \\
\hline DNAJB9 & -1.774 & $7.49 \mathrm{E}-49$ & $1.44 \mathrm{E}-46$ \\
\hline ST13 & -1.484 & $2.45 \mathrm{E}-46$ & $2.36 \mathrm{E}-44$ \\
\hline BIRC5 & 1.474 & $5.62 \mathrm{E}-45$ & $3.61 \mathrm{E}-43$ \\
\hline DIRAS3 & -1.867 & $2.79 E-39$ & $1.34 \mathrm{E}-37$ \\
\hline HGS & 1.599 & $9.39 \mathrm{E}-39$ & $3.62 E-37$ \\
\hline GAPDH & 1.628 & $1.77 \mathrm{E}-35$ & 4.27E-34 \\
\hline VAMP7 & -1.290 & $3.20 \mathrm{E}-30$ & $6.17 \mathrm{E}-29$ \\
\hline PEA15 & 1.065 & $1.02 \mathrm{E}-29$ & $1.60 \mathrm{E}-28$ \\
\hline CAPN1 & 1.165 & $1.08 \mathrm{E}-29$ & $1.60 \mathrm{E}-28$ \\
\hline CAPN2 & -1.263 & $6.24 \mathrm{E}-29$ & $8.60 \mathrm{E}-28$ \\
\hline PRKAR1A & -1.055 & $1.38 \mathrm{E}-27$ & $1.67 \mathrm{E}-26$ \\
\hline ARSA & 1.077 & $6.94 \mathrm{E}-26$ & $6.69 \mathrm{E}-25$ \\
\hline $\mathrm{P} 4 \mathrm{HB}$ & 1.387 & $1.09 \mathrm{E}-25$ & $9.98 \mathrm{E}-25$ \\
\hline VEGFA & 1.027 & $3.12 \mathrm{E}-25$ & $2.74 \mathrm{E}-24$ \\
\hline CDKN1B & -1.338 & $1.64 \mathrm{E}-24$ & $1.32 \mathrm{E}-23$ \\
\hline ATG2B & -1.581 & $3.71 \mathrm{E}-24$ & $2.75 E-23$ \\
\hline CXCR4 & 1.353 & $2.39 \mathrm{E}-23$ & $1.59 \mathrm{E}-22$ \\
\hline RAB33B & -1.291 & $6.16 \mathrm{E}-23$ & $3.72 \mathrm{E}-22$ \\
\hline GAA & 1.086 & $4.38 \mathrm{E}-22$ & $2.49 \mathrm{E}-21$ \\
\hline HSPA5 & 1.155 & $6.10 \mathrm{E}-22$ & $3.27 E-21$ \\
\hline PRKCD & 1.104 & $1.42 \mathrm{E}-21$ & $7.22 \mathrm{E}-21$ \\
\hline SIRT2 & 1.210 & $5.31 \mathrm{E}-19$ & $2.23 \mathrm{E}-18$ \\
\hline CAPNS1 & 1.574 & $6.48 \mathrm{E}-19$ & $2.66 \mathrm{E}-18$ \\
\hline CTSD & 1.250 & $1.68 \mathrm{E}-18$ & $6.47 \mathrm{E}-18$ \\
\hline PEX3 & -1.012 & 7.33E-18 & $2.57 \mathrm{E}-17$ \\
\hline ATG3 & -1.069 & $3.08 \mathrm{E}-16$ & $9.59 \mathrm{E}-16$ \\
\hline RB1 & -1.121 & $3.90 \mathrm{E}-15$ & $1.12 \mathrm{E}-14$ \\
\hline ElF4EBP1 & 1.002 & $2.87 \mathrm{E}-13$ & 7.39E-13 \\
\hline FKBP1B & -1.039 & $1.63 \mathrm{E}-12$ & $3.99 \mathrm{E}-12$ \\
\hline ITGB1 & -1.107 & 2.73E-12 & $6.42 \mathrm{E}-12$ \\
\hline HSP90AB1 & 1.028 & 4.50E-09 & 8.69E-09 \\
\hline
\end{tabular}

GO analysis shows that DEARGs are enriched in several hypoxia-related biological processes (BP), including autophagy, macroautophagy, response to hypoxia and response to oxygen levels. Regarding the molecular function (MF), the DEARGs played vital parts in some functions, such as ubiquitin-like protein ligase binding, chaperone binding and heat shock protein binding. In terms of the cellular components $(\mathrm{CC})$, the proteins encoded by the DEARGs are components of melanosome, pigment granule and secretory granule lumen (Fig. 2c). KEGG analysis showed the main pathways that DEARGs involved containing autophagy, and protein processing in the endoplasmic reticulum (Fig. $2 \mathbf{d}$ ).

\section{Establishment of autophagy-related signature}

Altogether RNA-seq and clinical data of 379 EOC tissue specimens were obtained from the TCGA database and 374 EOC tissue specimens contained complete clinical follow-up information were subjected to univariate Cox regression analyses to evaluate the association between 31 screened DEARGs' expression profiles and overall survival in 374 EOC patients. The results of univariate Cox regression analyses revealed that 9 DEARGs were significantly associated with the prognosis of EOC patients $(p<0.05)$ (Fig. 3a). To improve the validity and robustness, 9 prognostic-related DEARGs obtained from the above univariate analysis were further subjected to the multivariate Cox regression analysis. Finally, 5 DEAR Gs (PEX3, DNAJB9, RB1, HSP90AB1 and CXCR4) were screened out as optimal independent prognosis biomarkers and applied to construct an autophagy-related risk model (Fig. 3e). The risk score of OS for EOC patients was calculated according to the following formula: risk score $=(0.3719 \times$ expression value of $P E X 3)+$ $(-0.4819 \times$ expression value of $D N A J B 9)+(0.3235 \times$ expression value of $R B 1)+(-0.4574 \times$ expression value of $H S P 90 A B 1)+(-0.1754 \times$ expression value of CXCR4). The risk scores of 374 EOC patients were calculated through the above formula, and patients were divided into high-risk $(n=187)$ and low-risk group $(\mathrm{n}=$ 187 ) with the median of the risk score as the cutoff value.

\section{Validation of the risk signature}

The OncoPrint in cBioPortal is a compact and efficient graphical summary of genomic alterations in tumor 


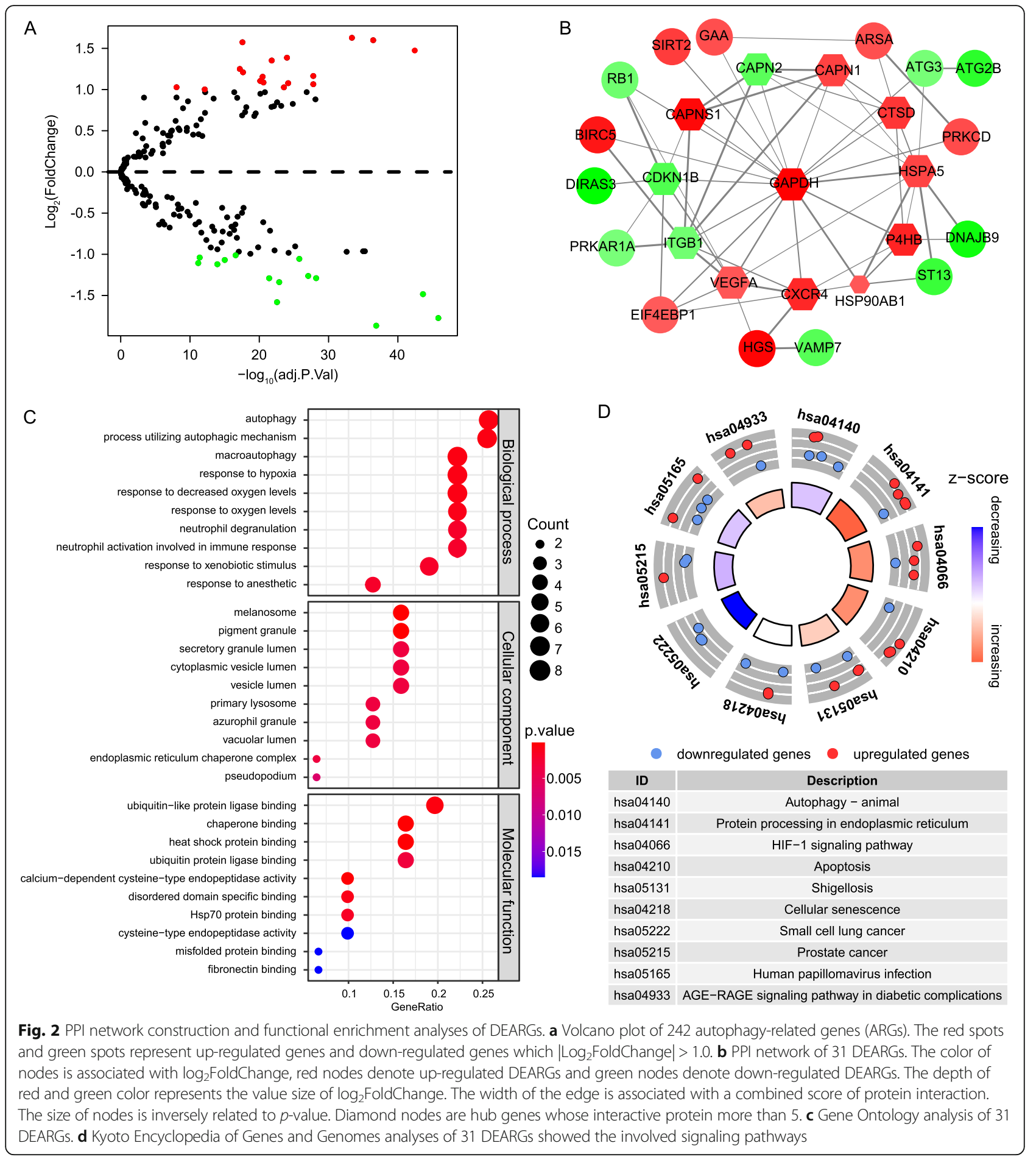

specimens. According to previous results of DEARGs screening and autophagy-related signature establishment, the risk score calculated based on the expression of PEX3, DNAJB9, RB1, HSP9OAB1 and CXCR4 can be a predictor of OS for EOC patients independent of other clinicopathological parameters. We analyzed and visualized genomic alterations of 5 prognostic DEARGs by
cBioPortal in ovary carcinoma cases (Fig. 4a). KaplanMeier plot was drawn to compare the OS difference between high-risk and low-risk group. EOC patients in the low-risk group had obviously better survival outcomes than the high-risk group ( $p=9.606 \mathrm{E}-07)$ illustrated that the risk score based on the autophagy-related risk model correlated with EOC patients' prognosis (Fig. 4b). 


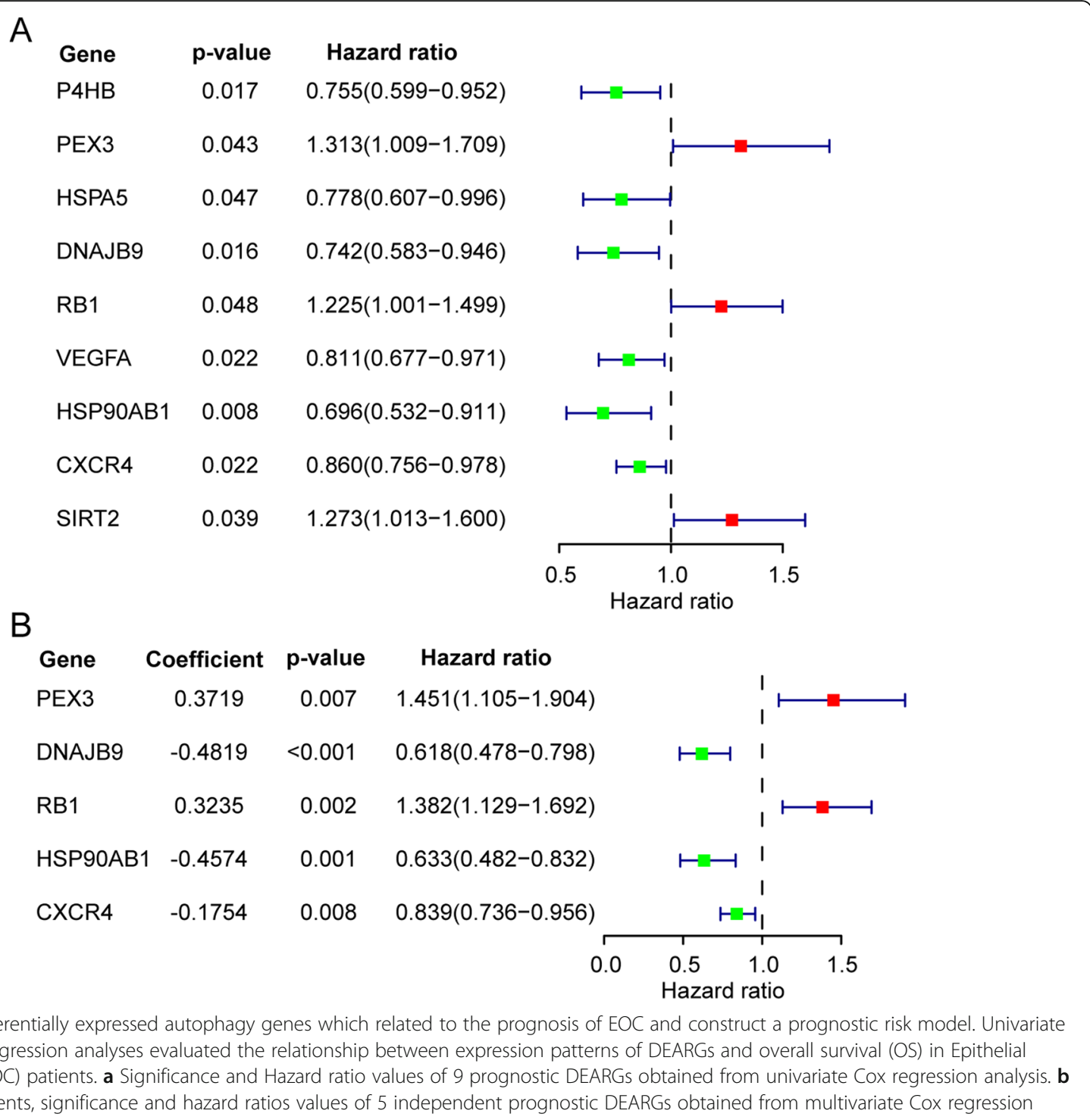

Figure.4c visualized the risk scores from low to high. The mortality of EOC patients increased as the risk score increased (Fig. 4d). The heatmap in Fig. 4e displayed the expression patterns of 5 prognostic DEARGs in different risk groups.

\section{Clinical utility of prognostic signature}

The univariate and multivariate Cox proportional hazard regression analyses were performed to determine the correlation between the risk score and clinicopathological features, and to further evaluated whether the autophagy-related risk prognostic model constructed with DEARGs could independent of other clinicopathological features as a predictor of OS for EOC patients. There is no difference of risk scores between age $>65$ and age $\leq 65(p=0.211)$ (Fig. 5a) or pathological stage IIIC-IV and pathological stage I-IIIB $(p=0.195)$ (Fig. 5b), although elder patients seemed had a higher risk score than younger patients and high pathological stage patients seemed had a higher risk score than low pathological stage patients. Risk score was higher in histological grade G3-4 than in G1-2 $(p=0.011)$ (Fig. 5c). Obviously, the majority of EOC patients are diagnosed at a late stage, so most patients are diagnosed with pathological stage III or histological grade 3-4. The association between the expression level of 5 prognostic DEARGs which used to construct the risk model and clinical pathological parameters of EOC are shown in Fig. 5 a, b, c.

In Table 3, univariate and multivariate Cox regression analysis showed that age and risk score was significantly correlated with OS of EOC, risk score can be an independent factor for the OS of EOC. These results confirmed that the autophagy-related prognostic signature 


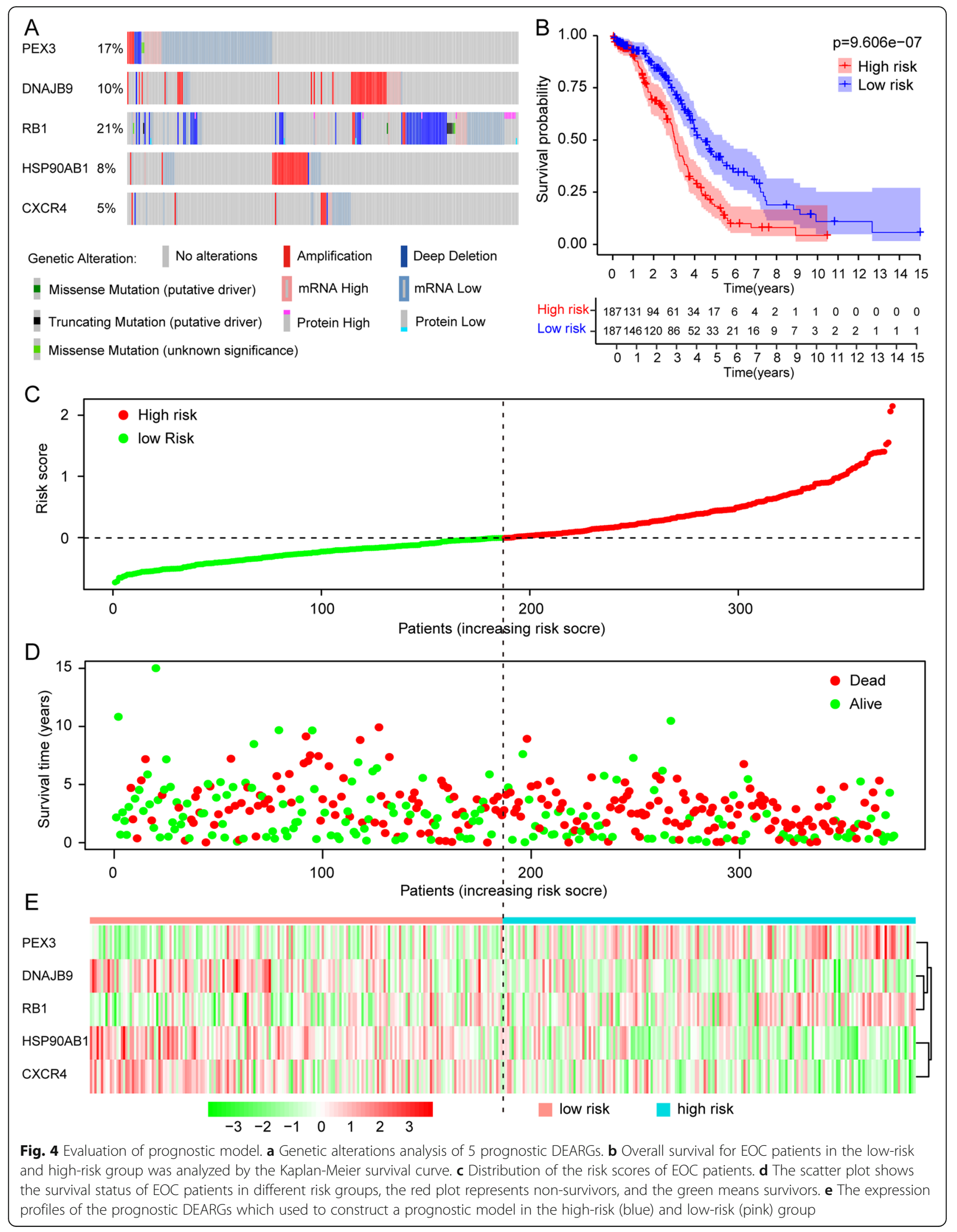




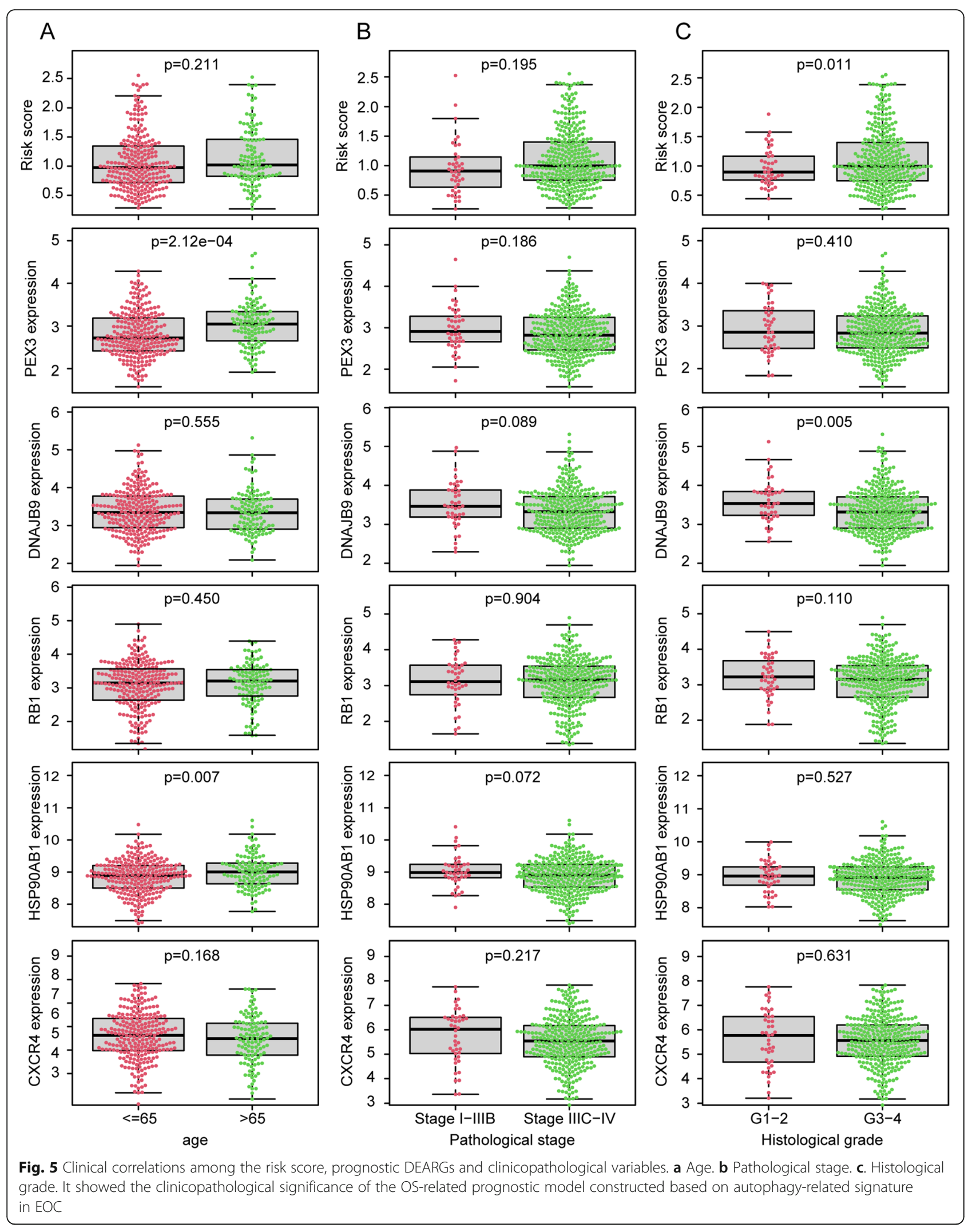


Table 3 Univariate and multivariate cox regression analyses of riskscore and clinicopathologic features in the TCGA group EOC patients

\begin{tabular}{|c|c|c|c|c|}
\hline \multirow[t]{2}{*}{ Variables } & \multicolumn{2}{|l|}{ Univariate analysis } & \multicolumn{2}{|c|}{ Multivariate analysis } \\
\hline & HR $(95 \% \mathrm{Cl})$ & $p$-Value & HR $(95 \% \mathrm{Cl})$ & $p$-Value \\
\hline RiskScore & $2.120(1.629-2.759)$ & $<0.001$ & $2.107(1.609-2.760)$ & $<0.001$ \\
\hline Age & $1.020(1.006-1.033)$ & 0.004 & $1.020(1.007-1.034)$ & 0.003 \\
\hline Pathological Stage & 1.353(0.999-1.832) & 0.051 & $1.300(0.947-1.786)$ & 0.105 \\
\hline Histological Grade & $1.448(0.968-2.165)$ & 0.071 & $1.244(0.828-1.868)$ & 0.293 \\
\hline
\end{tabular}

can be an independent indicator for prognosis monitoring for EOC patients.

\section{Discussion}

Autophagy is a eukaryotic cellular degradation and recycling process which is highly conserved [19]. Many studies had confirmed that autophagy plays a significant role in EOC [20-23]. Some pharmaceutical agents targeting autophagy have been proved effective in EOC patients $[24,25]$. Up to now, histological grade and stage remain the strongest prognostic evaluation tools in EOC [26]. Make use of a prognostic model which constructed based on the specific molecule can improve the understanding of the molecular mechanism of EOC, aid the development of more specific therapies, and identify novel biomarkers $[27,28]$. So, we construct autophagyrelated prognostic signature with DEARGs to predict the prognosis of EOC patients.

According to our study, an autophagy-related independent prognostic signature is constructed by 5 ARGs with different coefficients, including PEX3, DNAJB9, RB1, HSP9OAB1 and CXCR4. There were only a few researches reported a relationship between the 5 ARGs and cancers, Daniela et al and Shaobo et al reported PEX3 plays an important role in Melanoma [29] and colon cancer [30] respectively. DNAJB9 is a known negative feedback regulator of the tumor suppressor gene p53 in nongestational choriocarcinoma [31] and can reduce chemotherapy resistance in acute myeloid leukemia [32]. Guang et al reported that $R B 1$ is a tumor suppressor in OC [33]. $H S P 90 A B 1$ previously known as heat shock $90-\mathrm{kDa}$ protein 1 , beta, its expression is relatively stable in ovarian tissues [34]. As a chemokine receptor, blocking the CXCR4/ CXCL12 signal could be a potential therapy for EOC patients [35]. The results suggest that most autophagyrelated independent potential prognostic markers were identified and verified in other cancers except EOC, hence identifying potential prognostic markers and new therapeutic targets for EOC patients is essential.

There are 374 EOC patients' information with gene expression and survival data were obtained from TCGA to established an autophagy-related prognostic model, and only 364 patients with complete clinical information of age, pathological stage and histological grade. We verified the relationship between autophagy-related prognostic signature and their clinicopathological features. The 5 ARGs are not all associated with clinicopathological features of EOC patients, but the risk score calculated based on 5 prognostic DEARGs is significantly related to the histological grade of EOC patients. It is important to notice that among 364 EOC patients, only 43 patients were subjected to G1-G2, 321 patients were subjected to G3-G4. The same is only 22 patients diagnosed in stage I-II, the rest 342 patients all diagnosed in stage III-IV. Many patients diagnosed at an advanced stage disturbed the analysis for the relationship between the risk model and clinical information. Even so, we still can find a trend of risk score was higher in pathological stage IIIC-IV than in stage I-IIIB. Several assessment methods confirmed that the prognostic model can be an independent indicator for prognosis monitoring for EOC patients. Clinical information of EOC patients reminded us that early diagnosis is urgently needed for EOC patients. Our study shed light on finding the diagnosis and targeted treatment biomarkers in EOC patients.

Pathway enrichment analysis showed that 31 DEARGs were mainly involved in hypoxia or oxygen related pathways. Kalpana et al reported that hypoxia-induced carcinoma progression and metastasis, and drug resistance are serious problems for EOC treatment in clinical [36]. Edith et al proposed that $\mathrm{OC}$ metastasizes and recurs all in a unique hypoxia microenvironment in the abdominal cavity [37]. Hence, the affection of hypoxia to EOC patients should be evaluated and managed cautiously.

Our study developed a risk prognostic model to predict individuals' clinical outcomes, molecular signature combined with clinical features make the model steady and credible. Our results proved that the risk model constructed by 5 DEARGs PEX3, DNAJB9, RB1, HSP9OAB1 and CXCR4 is clinically practicable to evaluated prognosis for EOC patients. Besides traditional clinicopathological indicators (including pathological stage and histological grade), risk scores based on the autophagy-related genes signature could also be applied in clinical to provide a handy and better prognosis monitoring. The DEARGs can also help facilitate personalized target treatment and early screening. 


\section{Conclusions}

Our study analyzed transcriptome expression profiles of 354 EOC tissue samples and 56 HOSE samples from 7 independent laboratories and evaluated the expression pattern of 232 ARGs in the two groups. There were 17 up-regulated DEARGs and 14 down-regulated DEARGs in $\mathrm{EOC}$ with the filter criteria of adjusting $p$-value $<0.05$ and $\left|\log _{2} \mathrm{FC}\right|>1.0$. From 31 DEARGs, 5 prognostic DEARGs (PEX3, DNAJB9, RB1, HSP90AB1, CXCR4) were identified to construct a risk score prediction model, the risk score was calculated with the expression values of these genes and regression coefficients. Combined validation analysis of molecular signature and clinical characteristics, the risk score prediction model can robustly estimate the OS of EOC patients. The prognosis DEARGs also provide new possibilities for EOC therapeutic intervention. Meanwhile, our study can reveal the molecular mechanisms behind EOC from a brand-new insight.

\section{Abbreviations \\ ARGs: Autophagy- related genes; EOC: Epithelial ovarian cancer; DEAR Gs: Differentially expressed autophagy- related genes; HOSE: Human ovarian surface epithelia; GO: Gene Ontology; KEGG: Kyoto Encyclopedia of Genes and Genomes; KM plotter: Kaplan-Meier plotter; $\log _{2} \mathrm{FC}$ : $\log _{2}$ Fold Change/ Logarithm of Fold Change; OC: Ovarian cancer; OS: Overall survival; PPI: protein-protein interaction; TCGA: The Cancer Genome Atlas}

\section{Acknowledgements}

The authors apologize to those authors whose work could not be cited due to space limitations.

\section{Authors' contributions}

HF conceived of the study. HF and SC constructed and verified the prognostic model. HF performed the statistical analysis and drafted the manuscript. HF and SC performed cox analysis. CX supervised all the work and revised the manuscript.

\section{Funding}

The study was supported by the Nosocomial Scientific Research Fund Projects from the International Peace Maternity and Child Health Hospital of Shanghai Jiao Tong University School of Medicine (No.GFY5801), clinical research special projects from Shanghai Municipal Health Commission (No.20204Y0230) and Shanghai Sailing Program from Shanghai Science and Technology Committee (No.19YF1452200).

\section{Availability of data and materials}

The raw data supporting the conclusions of this manuscript will be made available by the authors, without undue reservation, to any qualified researcher.

\section{Declarations}

Ethics approval and consent to participate

Not applicable.

\section{Consent for publication}

Not applicable.

\section{Competing interests}

The authors have declared that no competing interest exists.
Received: 16 September 2020 Accepted: 26 February 2021

Published online: 06 March 2021

\section{References}

1. Momenimovahed Z, Tiznobaik A, Taheri S, Salehiniya H. Ovarian cancer in the world: epidemiology and risk factors. Int J Women's Health. 2019;11: 287-99.

2. Siegel RL, Miller KD, Jemal A. Cancer statistics, 2020. CA Cancer J Clin. 2020; 70(1):7-30.

3. Bray F, Ferlay J, Soerjomataram I, Siegel RL, Torre LA, Jemal A. Global cancer statistics 2018: GLOBOCAN estimates of incidence and mortality worldwide for 36 cancers in 185 countries. CA Cancer J Clin. 2018;68(6):394-424.

4. Gadducci A, Guarneri V, Peccatori FA, Ronzino G, Scandurra G, Zamagni C, Zola P, Salutari V. Current strategies for the targeted treatment of highgrade serous epithelial ovarian cancer and relevance of BRCA mutational status. J Ovarian Res. 2019;12(1):9.

5. Torre LA, Trabert B, DeSantis CE, Miller KD, Samimi G, Runowicz CD, Gaudet MM, Jemal A, Siegel RL. Ovarian cancer statistics, 2018. CA Cancer J Clin. 2018;68(4):284-96

6. Lheureux S, Gourley C, Vergote I, Oza AM. Epithelial ovarian cancer. Lancet. 2019;393(10177):1240-53.

7. Ezzati M, Abdullah A, Shariftabrizi A, Hou J, Kopf M, Stedman JK, Samuelson $\mathrm{R}$, Shahabi S. Recent advancements in prognostic factors of epithelial ovarian carcinoma. Int Scholarly Res Notices. 2014;2014:953509.

8. Chiang YC, Chen CA, Chiang CJ, Hsu TH, Lin MC, You SL, Cheng WF, Lai MS. Trends in incidence and survival outcome of epithelial ovarian cancer: 30year national population-based registry in Taiwan. J Gynecol Oncol. 2013; 24(4):342-51.

9. Ayhan A, Gultekin M, Celik NY, Dursun P, Taskiran C, Aksan G, Yuce K. Occult metastasis in early ovarian cancers: risk factors and associated prognosis. Am J Obstet Gynecol. 2007;196(1):81 e81-86.

10. Wahner Hendrickson AE, Hawthorne KM, Goode EL, Kalli KR, Goergen KM, Bakkum-Gamez JN, Cliby WA, Keeney GL, Visscher DW, Tarabishy Y, et al. Assessment of published models and prognostic variables in epithelial ovarian cancer at Mayo Clinic. Gynecol Oncol. 2015;137(1):77-85.

11. Bodelon C, Killian JK, Sampson JN, Anderson WF, Matsuno R, Brinton LA, Lissowska J, Anglesio MS, Bowtell DDL, Doherty JA, et al. Molecular classification of epithelial ovarian Cancer based on methylation profiling: evidence for survival heterogeneity. Clin Cancer Res. 2019;25(19):5937-46.

12. Xie H, Hou Y, Cheng J, Openkova MS, Xia B, Wang W, Li A, Yang K, Li J, Xu $\mathrm{H}$, et al. Metabolic profiling and novel plasma biomarkers for predicting survival in epithelial ovarian cancer. Oncotarget. 2017;8(19):32134-46.

13. Giampieri F, Afrin S, Forbes-Hernandez TY, Gasparrini M, Cianciosi D, Reboredo-Rodriguez P, Varela-Lopez A, Quiles JL, Battino M. Autophagy in human health and disease: novel therapeutic opportunities. Antioxid Redox Signal. 2019;30(4):577-634.

14. Levine B, Kroemer G. Biological functions of autophagy genes: a disease perspective. Cell. 2019;176(1-2):11-42.

15. Orfanelli T, Jeong JM, Doulaveris G, Holcomb K, Witkin SS. Involvement of autophagy in cervical, endometrial and ovarian cancer. Int J Cancer. 2014; 135(3):519-28

16. Liu Y, Tang J, Liu D, Zhang L, He Y, Li J, Gao L, Tang D, Jin X, Kong D. Increased autophagy in EOC re-ascites cells can inhibit cell death and promote drug resistance. Cell Death Dis. 2018:9(4):419.

17. Peracchio C, Alabiso O, Valente G, Isidoro C. Involvement of autophagy in ovarian cancer: a working hypothesis. J Ovarian Res. 2012;5(1):22.

18. Wang Q, Bu S, Xin D, Li B, Wang L, Lai D. Autophagy is indispensable for the self-renewal and quiescence of ovarian Cancer spheroid cells with stem cell-like properties. Oxidative Med Cell Longev. 2018:7010472.

19. Parzych KR, Klionsky DJ. An overview of autophagy: morphology, mechanism, and regulation. Antioxid Redox Signal. 2014;20(3):460-73.

20. Thuwajit C, Ferraresi A, Titone $R$, Thuwajit $P$, Isidoro $C$. The metabolic cross-talk between epithelial cancer cells and stromal fibroblasts in ovarian cancer progression: autophagy plays a role. Med Res Rev. 2018; 38(4):1235-54.

21. Wahba J, Natoli M, Whilding LM, Parente-Pereira AC, Jung Y, Zona S, Lam EW, Smith JR, Maher J, Ghaem-Maghami S. Chemotherapy-induced apoptosis, autophagy and cell cycle arrest are key drivers of synergy in chemo-immunotherapy of epithelial ovarian cancer. Cancer Immunol Immunother. 2018;67(11):1753-65. 
22. Gao L, Wang Z, Lu D, Huang J, Liu J, Hong L. Paeonol induces cytoprotective autophagy via blocking the Akt/mTOR pathway in ovarian cancer cells. Cell Death Dis. 2019;10(8):609.

23. Pagotto A, Pilotto G, Mazzoldi EL, Nicoletto MO, Frezzini S, Pasto A, Amadori A. Autophagy inhibition reduces chemoresistance and tumorigenic potential of human ovarian cancer stem cells. Cell Death Dis. 2017;8(7): e2943.

24. Tang J, Zhu J, Ye Y, Liu Y, He Y, Zhang L, Tang D, Qiao C, Feng X, Li J, et al. Inhibition LC3B can increase chemosensitivity of ovarian cancer cells. Cancer Cell Int. 2019;19:199.

25. Kumar A, Singh UK, Chaudhary A. Targeting autophagy to overcome drug resistance in cancer therapy. Future Med Chem. 2015;7(12):1535-42.

26. Arora N, Talhouk A, McAlpine JN, Law MR, Hanley GE. Long-term mortality among women with epithelial ovarian cancer: a population-based study in British Columbia, Canada. BMC Cancer. 2018;18(1):1039.

27. Leong HS, Galletta L, Etemadmoghadam D, George J, Australian ovarian Cancer S, Kobel M, Ramus SJ, Bowtell D. Efficient molecular subtype classification of high-grade serous ovarian cancer. J Pathol. 2015;236(3):272-7.

28. Cook DP, Vanderhyden BC. Ovarian cancer and the evolution of subtype classifications using transcriptional profilingdagger. Biol Reprod. 2019;101(3): 645-58.

29. D'Arcangelo D, Giampietri C, Muscio M, Scatozza F, Facchiano F, Facchiano A. WIPI1, BAG1, and PEX3 autophagy-related genes are relevant melanoma markers. Oxidative Med Cell Longev. 2018:1471682.

30. Mo S, Dai W, Xiang W, Li Y, Feng Y, Zhang L, Li Q, Cai G. Prognostic and predictive value of an autophagy-related signature for early relapse in stages I-III colon cancer. Carcinogenesis. 2019;40(7):861-70.

31. Lazare C, Zhi W, Dai J, Cao C, Sookha RR, Wang L, Meng Y, Gao P, Wu P, Wei J, et al. A pilot study comparing the genetic molecular biology of gestational and non-gestational choriocarcinoma. Am J Transl Res. 2019; 11(11):7049-62.

32. Wang D, Zeng T, Lin Z, Yan L, Wang F, Tang L, Wang L, Tang D, Chen P, Yang M. Long non-coding RNA SNHG5 regulates chemotherapy resistance through the miR-32/DNAJB9 axis in acute myeloid leukemia. Biomed Pharmacother. 2020;123:109802.

33. Peng G, Mills GB. Surviving ovarian Cancer: an affair between defective DNA repair and RB1. Clin Cancer Res. 2018:24(3):508-10.

34. Nikishin DA, Filatov MA, Kiseleva MV, Bagaeva TS, Konduktorova W, Khramova YV, Malinova IV, Komarova EV, Semenova ML. Selection of stable expressed reference genes in native and vitrified/thawed human ovarian tissue for analysis by qRT-PCR and Western blot. J Assist Reprod Genet. 2018;35(10):1851-60.

35. Mao TL, Fan KF, Liu CL. Targeting the CXCR4/CXCL12 axis in treating epithelial ovarian cancer. Gene Ther. 2017;24(10):621-9.

36. Dorayappan KDP, Wanner R, Wallbillich JJ, Saini U, Zingarelli R, Suarez AA, Cohn DE, Selvendiran K. Hypoxia-induced exosomes contribute to a more aggressive and chemoresistant ovarian cancer phenotype: a novel mechanism linking STAT3/Rab proteins. Oncogene. 2018:37(28):3806-21.

37. Emmings E, Mullany S, Chang Z, Landen CN Jr, Linder S, Bazzaro M. Targeting Mitochondria for Treatment of Chemoresistant Ovarian Cancer. Int J Mol Sci. 2019;20(1):229.

\section{Publisher's Note}

Springer Nature remains neutral with regard to jurisdictional claims in published maps and institutional affiliations.

Ready to submit your research? Choose BMC and benefit from:

- fast, convenient online submission

- thorough peer review by experienced researchers in your field

- rapid publication on acceptance

- support for research data, including large and complex data types

- gold Open Access which fosters wider collaboration and increased citations

- maximum visibility for your research: over $100 \mathrm{M}$ website views per year

At $\mathrm{BMC}$, research is always in progress.

Learn more biomedcentral.com/submissions 